\title{
Discovery of an Anomalous Non-contact Effect with a Pyramidal Structure
}

\section{Osamu Takagi ${ }^{1}$, Masamichi Sakamoto², Hideo Yoichi' ${ }^{1}$, Hideyuki Kokubo', Kimiko Kawano', Mikio Yamamoto ${ }^{1}$}

\author{
${ }^{1}$ Information and Research Center, International Research Institute (IRI), 1108-2 Sonno, Inage, Chiba 263- \\ 0051, Japan \\ ${ }^{2}$ Aquavision Academy, 1228-3 Tsubuura, Narita, Chiba 287-0236, Japan
}

\begin{abstract}
In 2013, we reported the existence of a meditator's non-contact effect toward edible cucumber slices used as bio-sensors with which the meditator had no direct physical contact. In the present paper, we investigated the long-term change of this anomalous non-contact effect. We discovered that the effect is associated with a pyramidal structure. We also discovered with extremely high reliability a new phenomenon an "anomalous non-contact effect with a delay associated with a pyramidal structure" in which the meditator's non-contact action creates a delayed effect over an extended period of time (10 or more days) after the non-contact action took place. We were able to approximate this anomalous non-contact effect with the delay by a mathematical formula describing a transient response phenomenon of the second order lag element system.
\end{abstract}

Keywords: Meditator, Non-Contact Effect, Bio-Sensor, Cucumber, Pyramidal Structure, Delayed Effect

\section{Introduction}

Unexplained functions which a pyramidal structure are said to have are of great scientific interest. On the other hand, although there have been various traditions and books which describe these functions, there have been almost no reliable academic studies and no statistically significant data about them in relation to the pyramidal structure.

Also, it is a scientifically interesting theme whether or not humans can affect plant samples without coming into physical contact with them. Almost no research has been conducted which scientifically measures this effect except by Grad (1976). Yamamoto et al. (2000, 2002) have conducted scientific research projects including the above theme, beginning at the National Institute of Radiological Sciences (NIRS) of Japan in 1995. They have done measurements of bio-photons (very low intensity visual range light emitted from bio-samples (Cohen and Popp 1997; Inaba 1997)) for fundamental experiments to utilize them as bio-sensors since 1998 (Parkhomtchouk et al. 2000). In 2005 Yamamoto's group moved from NIRS to the International Research Institute (IRI). Since then at IRI, the group has developed a bio-photon method, a fluorescence method and a gas method which utilize edible cucumber slices as bio-sensors (Kokubo et al. 2006; Kokubo et al. 2009; Kokubo et al. 2012). The gas method, which measures 2-hexanol gas emitted from cucumber slices with gas detecting tubes, enables measurement of a number of samples simultaneously at low cost. Therefore, we used that method in this paper.

In 2013, we scientifically proved one example which shows a non-contact effect toward bio-samples with very high statistical reliability $\left(\mathrm{p}=3.13 \times 10^{-10}\right.$, t-test, two-tails) by strict experiments and an analysis method as part of the Sakamoto Hyper-Tech Project (SHyP) carried out in IRI (Takagi et al. 2013). As a result of comparing the "presence" (the "meditation period") of the meditator with the "absence" (the "before and after meditation periods") of the meditator strictly using the gas method, we were able to obtain this surprising experimental result.

Our purpose for the present paper is to prove the following phenomenon by strict experiments and analysis: Through the intervention of a pyramidal structure (PS), a meditator's non-contact action has a prolonged non-contact effect toward the bio-sensors. Here, the meditator and the bio-sensors are physically separated and the PS is a simple and static structure which does not have any mechanically movable parts 
nor does it have a supply of known energy. That is, our purpose is to prove scientifically a completely new phenomenon an "anomalous non-contact effect with a delay associated with a pyramidal structure" in which a non-contact effect is detected over a long period of time (10 or more days) after the non-contact action took place.

\section{Experimental Method}

We used the gas method in order to investigate the non-contact effect on cucumber slices (Kokubo et al. 2009).

\section{2-1.Pyramidal structure}

The Pyramidal structure (PS) used was a squarebased pyramid with a height of $107 \mathrm{~cm}$, a ridgeline length of $170 \mathrm{~cm}$ and a base length of $188 \mathrm{~cm}$. The frame was made of four aluminum pipes $(2 \mathrm{~cm}$ diameter, $0.36 \mathrm{~cm}$ thick pipe wall), the top ends of which were connected. The bottom ends of each pipe were placed at the four corners of a square $(188 \mathrm{~cm}$ on each side) made with four aluminum L angles. For the meditator to be able to go inside the PS, the base of the PS was raised by four tripods $73 \mathrm{~cm}$ from the floor. The four aluminum pipes were not electrically connected to the L angles, and they were not electrically grounded. The four sides of the PS were made of $1 \mathrm{~cm}$ thick polystyrene boards, which were not grounded. Each side had a Sierpinski triangle pattern with $\mathrm{n}=4$ and it consisted of nine aluminum plates $(40.85 \mathrm{~cm} \times 40.85 \mathrm{~cm} \times 43 \mathrm{~cm}, 0.3 \mathrm{~mm}$ thick) which were attached to the polystyrene board. Further, each aluminum plate had a Sierpinski triangle pattern with $n=2$. The four aluminum pipes were electrically connected to the aluminum plates on all the sides, but since the pipes were not grounded, the aluminum plates were not either. We call this PS a fractal pyramid (Takagi et al. 2013). Inside the PS, a transparent acrylic $(0.5 \mathrm{~cm}$ thick $)$ dome $(85 \mathrm{~cm}$ diameter, $66.5 \mathrm{~cm}$ high) with a spherical shell shape was placed. The lower portion of the sphere had been removed where the diameter of the sphere cross section was $68.1 \mathrm{~cm}$. The dome was sitting on a wooden square board $(99 \mathrm{~cm} \times 99 \mathrm{~cm}, 3 \mathrm{~cm}$ thick) with a $70 \mathrm{~cm}$ diameter hole in the center that allowed the meditator to insert his head and upper body into the dome space. The bottom surface of the board was held at a height of $85 \mathrm{~cm}$ by four tripods. The dome was designed in such a way that the meditator's voice resonated inside.

\section{2-2. Meditation method}

The meditator (a male with 22 years of Hemi-Sync ${ }^{\circledR}$ (Atwater 1997) experience) was the same person as had participated in the previous experiment (Takagi et al. 2013), Masamichi Sakamoto. In relation to participation in the meditation, his written consent had been obtained. The meditation was performed as follows. The meditator sat on a chair which was placed directly below the PS apex. He performed three meditation runs in the morning and another three runs in the afternoon, each run being $30 \mathrm{~min}$. During the meditation, he listened to Hemi-Sync $\AA$, which is an audio technology designed to help the listener to meditate for an extended period of time. The meditator wore a set of stereo headphones so that there was no sound leakage to the environment. He sometimes chanted. He did not focus his attention on the cucumber slices which were placed right above him. After the meditation run, he wrote down its contents.

\section{2-3. Gas measurement}

We used the gas method in order to investigate the non-contact effect on cucumber slices (Kokubo et al. 2009). Following the simultaneous calibration technique (SCAT) (Kokubo et al. 2011), four pairs of an experimental sample dish and its cover (EXP) and a control sample dish and its cover (CONT) were prepared. A randomly selected identification label was placed into each dish. Two pairs were randomly chosen as the main samples (EXP1, CONT1, EXP2, CONT2), while the other two pairs became control samples (EXP3, CONT3, EXP4, CONT4).The EXP1 and EXP2 were placed $0.2 \mathrm{~cm}$ above the PS peak with an insulating supporting material, while the CONT1 and CONT2 were placed on the Calibration Control Point $8 \mathrm{~m}$ away from the PS. All the control samples were placed on the Calibration Control Point Both the main and the control samples were inside electrostatically shielded Faraday cages.

Each sample was placed at its point for $30 \mathrm{~min}$. Immediately afterward, all of them were collected, the covers were removed and each dish was placed within a sealable polypropylene container. All the containers were stored in a temperature controlled room (at $22-24{ }^{\circ} \mathrm{C}$ ) with no direct sunlight. The place where they were stored was on average $4 \mathrm{~m}$ away from the center of the PS. The concentration in the container became stable in 12-15 hs (Kokubo et al. 2010). After they were stored for a minimum of $36 \mathrm{~h}$, the concentration of 2-hexanol gas for the EXP and the CONT containers was measured at the same time (gas sampling pump, GV-100, Gastech, Japan; gas detecting tubes, 141L, Gastech, Japan). We have described the case in which four paired sample dishes are prepared from four cucumbers. We call this one run. In the real experiment, 3 runs of 4 paired sample dishes, i.e. 12 paired sample dishes in total were prepared at the same time using 12 cucumbers.

\section{2-4. Calibrated J value}

In order to investigate the strength of the non-contact effect that the meditator's action has, which is transmitted through the PS without direct physical contact to the bio-sensors, we employed $\mathbf{J}$ value. J value is obtained as a result of calculating the natural logarithm of the ratio of the 2-hexanol gas 
concentration measured in each pair of containers (EXP and CONT).

$$
J=k \ln \left(\frac{C_{E X P}}{C_{C O N T}}\right)
$$

Here, $\mathrm{k}$ is a proportionality coefficient $(\mathrm{k}=1)$ (Kokubo et al. 2010). $\mathrm{C}_{\mathrm{EXP}}$ and $\mathrm{C}_{\mathrm{CONT}}$ are the gas concentrations in a container containing EXP and CONT. If the environment of EXP and CONT are equivalent, the detected values of $\mathrm{C}_{\mathrm{EXP}}$ and $\mathrm{C}_{\mathrm{CONT}}$ are equal and thus $\mathrm{J}=0$. In each pair, $\mathrm{J}$ value calculated from the main samples (EXP1, CONT1 and EXP2, CONT2) is $\mathrm{J}_{\mathrm{E}}$, while $\mathrm{J}_{\mathrm{CAL}}$ is calculated from the control samples (EXP3, CONT3 and EXP4, CONT4). $\mathrm{J}_{\mathrm{E}-\mathrm{CAL}}$ is introduced in order to calculate the calibrated value.

$$
J_{E-C A L}=J_{E}-J_{C A L} \text { (2) }
$$

The value of $\mathrm{J}_{\mathrm{E}-\mathrm{CAL}}$ can be considered to correspond to the strength of the non-contact effect.

\section{2-5. Meditation experiment}

Figure 1 shows the concept for the meditation experiment (ME). The ME consisted of six parts done on the day when the meditator came to the lab and meditated. The six parts were: pre-1(-16h), pre-2($3 \mathrm{~h})$, med-1(0h), med-2(3h), post-1(5h) and post$2(20 \mathrm{~h})$. Here, the "before meditation period" (pre-1($16 \mathrm{~h})$, pre-2(-3h)) and the "after meditation period" (post-1(5h), post-2(20h)) corresponded to the four parts when the meditator was absent, whereas the "meditation period" (med-1(0h), med-2(3h)) corresponded to the two parts when he was present. In both the "before and after meditation periods", the meditator was at least $5 \mathrm{~km}$ away from the ME site.

Figure 2(a) shows the sequence of the six parts from pre-1(-16h) to post-2(20h) with the horizontal axis being time. We set the beginning of the med-1(0h), which was 10:00am, as the standard time 0 . Thus, in the case of pre-1(-16h), the experiment started $16 \mathrm{~h}$ before the standard time, pre-2(-3h) $3 \mathrm{~h}$ before, med2 (3h) $3 \mathrm{~h}$ after, post-1(5h) $5 \mathrm{~h}$ after, and post-2(20h) $20 \mathrm{~h}$ after the standard time.

Each part in the "before and after meditation periods" was composed of three runs. In the 1st run, the EXP1 and EXP2 were placed $0.2 \mathrm{~cm}$ above the PS top, while the CONT1 and CONT2 were placed on the Calibration Control Point. All the control samples were placed on the Calibration Control Point. All sample dishes were kept at their respective positions for $30 \mathrm{~min}$. Then after the lids were removed, the uncovered dishes were placed within containers and stored. $10 \mathrm{~min}$ after the end of the 1 st run, the 2 nd run was done by the same procedure. Then, $10 \mathrm{~min}$ afterward, the 3rd run was done. Thus three runs were obtained for one part. Each part in the "meditation period" (med-1(0h), med-2(3h)) also had three runs, and these parts differed from the "before and after meditation periods" runs only in that the meditator was inside the PS for $30 \mathrm{~min}$. The meditator had a lunch break between med-1(0h) and med-2(3h). In one ME, we used 72 cucumbers, 144 petri dishes and 144 gas detecting tubes.

\section{2-6 Non-meditation experiment}

Figure 2(b) shows the sequence for the nonmeditation experiment (NME); the meditator was not present during the day when this was done. In order to investigate the long term trend of the non-contact effect, the NME was performed a few days after and about 20 days after the standard time 0 . In the NME, six parts which corresponded to the six parts of the ME (pre-1(-16h) to post-2(20h)) were performed with the same timing and sequence as the ME.

\section{Experimental Results for the ME and NME}

Figure 3 shows the results for the ME, which consisted of "meditation period" (med-1(0h), med$2(3 \mathrm{~h}))$ and "after meditation period" (post-1(5h), post-2(20h)) and also the results for the NME, done a few days after the standard time 0 .

The colored symbols indicate the difference in the daytime length of the day the cucumbers were harvested. Assuming the harvest day to be four days before the beginning of the ME or the NME, the red diamond data correspond to cucumbers harvested when the daytime length (the length from sunrise to sunset in Tokyo) was longer than $12 \mathrm{~h}$ and we call them the summer data. The blue square data correspond to cucumbers harvested when the daytime length was shorter than $12 \mathrm{~h}$ and we call them the winter data.

For the summer data, the detected value $\left(\mathrm{J}_{\mathrm{E}-\mathrm{CAL}}\right)$ for the "meditation period" (med-1(0h), med-2(3h)) was 0 within the margin of error, indicating that the noncontact effect was not detected. However, starting at $5 \mathrm{~h}$ after the standard time 0 (post-1(5h)), $\mathrm{J}_{\mathrm{E}-\mathrm{CAL}}$ began to increase and reached the maximum $20 \mathrm{~h}$ after 0 (post-2(20h)). Then it decreased exponentially over an extended period of time, becoming almost zero 20 days after the standard time 0 .

This indicates that we have discovered a new amazing phenomenon in which a non-contact effect shows a delay over an extended period of time, longer than 10 days after the beginning of meditation.

Approximately 20 days after the meditation, $\mathrm{J}_{\mathrm{E}-\mathrm{CAL}}$ was not detected, indicating that the top of the PS had returned to a state like that which it had initially had. From this fact, we decided to call the NME which was done when there had not been a meditator in the PS for the preceding 20 days or longer a pure nonmeditation experiment (p-NME) (Fig. 2 (b)). 
It is important that the top of the PS has returned to its initial state each time we conduct a ME in order to have reliability. Therefore, the MEs should be performed at an interval of 20 days or longer.

In contrast, in the case of the winter data in Fig. 3, $\mathrm{J}_{\mathrm{E}-}$ CAL was zero within the margin of error for all the data points, i.e. med-1(0h), med-2(3h), post-1(5h) and post-2(20h) and also NMEs (6 and 14 days after the standard time) and the p-NME (19.5days after the standard time). Thus, the non-contact effect was not detected.

The inset of Fig. 3 shows the results of four parts, med-1(0h), med-2(3h), post-1(5h) and post-2(20h) for both the ME and the p-NME, which correspond to the expansion of Fig. 3 in the range of $0-20 \mathrm{~h}$. The black circle data were the p-NME data which correspond to one part from med-1(0h) to post$2(20 \mathrm{~h})$, and for these data all $\mathrm{J}_{\mathrm{E}-\mathrm{CAL}}$ values were zero within the margin of error, indicating that the noncontact effect was not detected. As we have explained, this reflected the result that the noncontact effect was not detected when there had been no meditator inside the PS for longer than 20 days. By comparing the p-NME data with the ME summer data for post-1(5h), which corresponds to the time when there was no meditator, we were able to prove the existence of the non-contact effect associated with the PS with very high statistical reliability $\left(\mathrm{p}=3.51 \times 10^{-6}\right.$, Welch's t-test, two-tails).

In Fig. 3, "meditation period" (med-1(0h), med$2(3 \mathrm{~h})$ ) data and "after meditation period" (post-1(5h), post-2(20h)) data were averages of the data for each part. In contrast, in the NME and p-NME, the averages of all six parts from pre-1(-16h) to post$2(20 \mathrm{~h})$ were plotted. The reason behind this was that in the case of the NME and the p-NME, there was no time dependence in $\mathrm{J}_{\mathrm{E}-\mathrm{CAL}}$.

For the "before meditation period" data (pre-1(-16h), pre-2(-3h)), although we obtained an upward trend from pre-1(-16h) to pre-2(-3h) regarding $\mathrm{J}_{\mathrm{E}-\mathrm{CAL}}$, it is too early to tell whether or not there is a non-contact effect in the "before meditation period". More investigation is necessary, which is now under way.

The number of gas measurements performed for this paper was 2,896 in total, among which 1,584 were for the med-1(0h), med-2(3h) and post-1(5h) parts of the ME. These 1,584 data included 504 data which had been used in the previous paper (Takagi et al. 2013). The data for the present paper were taken during the period from June 2011 to April 2013.

\section{Theoretical consideration}

The summer data in Fig. 3 indicate that the meditator's action created a non-contact effect for 10 or more days through the intervention with the PS.
This non-contact effect is considered to result from the conversion process of the meditator's non-contact action. The conversion process is inferred to be the following two-step reaction.

1. The meditator's action (input) is accumulated in the PS, and it is converted into another action.

2. The converted action (discharge) is detected by the bio-sensors.

We have assumed that the meditator's non-contact action is converted into another action because the non-contact effect toward the bio-sensors was not detected in the "meditation period" (med-1(0h), med$2(3 \mathrm{~h})$ ), when the meditator's action was taking place. And this two-step reaction is considered to be a phenomenon corresponding to the transient response model of the second order lag element system in which the system shifts from a steady state to an excited state, and then shifts back to the steady state again.

We call the meditator's non-contact action toward the PS the input signal $u(t)$. And we call the response function $\mathrm{g}(\mathrm{t})$, in the case of $\mathrm{u}(\mathrm{t})$ being a delta function $\delta(\mathrm{t})$. Then, the output signal $\mathrm{y}(\mathrm{t})$ is written as follows.

$$
y(t)=\int_{0}^{t} g(t-\tau) u(\tau) d \tau=L^{-1}[G(s) U(s)]
$$

Here, $\mathrm{G}(\mathrm{s})=\mathrm{L}[\mathrm{g}(\mathrm{t})], \mathrm{U}(\mathrm{s})=\mathrm{L}[\mathrm{u}(\mathrm{t})], \mathrm{L}$ is a Laplace transformation, and $\mathrm{L}^{-1}$ is a Laplace inverse transformation.

The value of $y(t)$ is considered to correspond to $\mathrm{J}_{\mathrm{E}-\mathrm{CAL}}$ which shows the strength of the non-contact effect toward the bio-sensors. As a result, the change of $\mathrm{J}_{\mathrm{E}-}$ CAL corresponds to the transient response of the output signal $\mathrm{y}(\mathrm{t})$, when the meditator's non-contact action is the input signal $u(t)$.

Generally, the transfer function G(s) of the second order lag element system is written as follows.

$$
G(s)=\frac{K \omega_{n}^{2}}{s^{2}+2 \xi \omega_{n} s+\omega_{n}^{2}}
$$

Here, $\xi$ is a damping coefficient, $\omega_{n}$ is specific angular frequency, and $\mathrm{K}$ is gain constant.

Now we set

$\xi=\frac{\tau_{1}+\tau_{2}}{2 \sqrt{\tau_{1} \tau_{2}}}, \quad \omega_{n}=\frac{1}{\sqrt{\tau_{1} \tau_{2}}}$.

Then, eq. (4) becomes

$$
G(s)=\frac{K}{\left(\tau_{1} s+1\right)\left(\tau_{2} s+1\right)} .
$$

Equation (6) is equivalent to the transfer function of the second order lag element system in which two primary system elements $G(s)=\frac{1}{\tau_{1} s+1} \quad$ and 
$G(s)=\frac{K}{\tau_{2} s+1}$ are coupled in series. Here, $\tau_{1}$ and

$\tau_{2}$ are time constants. We note that eq. (6) will not change its form by switching $\tau_{1}$ with $\tau_{2}$, keeping the value of the output $y(t)$ the same.

It is not possible to identify precisely the function form of the input signal $u(t)$ which is the meditator's non-contact action on the PS. However, we can assume a reasonable form as the $\mathrm{u}(\mathrm{t})$ and calculate the output $y(t)$ to compare it with the experimental results. First, the output y(t) is described by eq. (7) when the input signal which acts on the PS is a delta function $\mathrm{A}_{0} \delta(\mathrm{t}),\left(\mathrm{A}_{0}=1\right)$.

$$
y(t)=\frac{A_{0} K}{\tau_{1}-\tau_{2}}\left\{e^{-\frac{t}{\tau_{1}}}-e^{-\frac{t}{\tau_{2}}}\right\}
$$

Next, we calculate the output $y(t)$ for the actual case in which the input signal $u(t)$ reproduces the actual pattern in the "meditation period" of med-1(0h) and med-2(3h). The form of the input signal $\mathrm{u}(\mathrm{t})$ is shown in the inset of Fig. 4. The $u(t)$ consists of six rectangular waves. We assume that the PS received a constant amount of action during the 30 minute meditation. A is calculated to be equal to 8 when the total amount of the action of the six meditation runs (two periods with three runs each) is set to be one unit.

The output $y(t)$ can be written as follows when the incoming signal $\mathrm{u}(\mathrm{t})$ is as shown in the inset of Fig. 4 .

$$
y(t)=\frac{A K}{\tau_{1}-\tau_{2}}\left\{-\sum_{n=1}^{6} F_{n}(t)+\sum_{n=7}^{12} F_{n}(t)\right\}
$$

$$
\text { where } F_{n}(t)=\left\{\tau_{1} e^{-\frac{1}{\tau_{1}}\left(t-t_{n}\right)}-\tau_{2} e^{-\frac{1}{\tau_{2}\left(t-t_{n}\right)}}-\left(\tau_{1}-\tau_{2}\right)\right\} f\left(t-t_{n}\right)
$$

Here,

$$
\begin{aligned}
& t_{1}=0, \quad t_{2}=a+b, \quad t_{3}=2 a+2 b, \quad t_{4}=3 a+2 b+c, \quad t_{5}=4 a+3 b+c, \quad t_{6}=5 a+4 b+c, \\
& t_{7}=a, \quad t_{8}=2 a+b, \quad t_{9}=3 a+2 b, \quad t_{10}=4 a+2 b+c, \quad t_{11}=5 a+3 b+c, \quad t_{12}=6 a+4 b+c .
\end{aligned}
$$

$\mathrm{f}(\mathrm{t})$ is a unit step function.

Figure 4 plots the calculation results of eq. (8) together with the summer data from Fig. 3. Here, $\mathrm{A}=8, \mathrm{~K}=1.22,\left(\tau_{1}, \tau_{2}\right)=(4.65,0.13)$ or $(0.13,4.56)$. The output $\mathrm{y}(\mathrm{t})$ approximates very well the experimental data of the anomalous non-contact effect with a delay. Moreover, it is clearly shown that the $y(t)$ corresponds to a transient response of the second order lag element system with a damping coefficient $\xi=3.074$, a specific angular frequency $\omega_{\mathrm{n}}=1.286$ and a gain coefficient $\mathrm{K}=1.22$.

\section{Results and discussion}

In the "meditation period", the non-contact effect on bio-sensors was not detected, whereas it was detected in the "after meditation period". It was detected in post-1(5h) which was performed $5 \mathrm{~h}$ after the start of the meditation. The detected value reached the maximum about $20 \mathrm{~h}$ after the start of the meditation. It declined exponentially after the maximum, and was detectable for more than 10 days and became zero within the margin of error about 20 days after the start of the meditation. We named this surprising phenomenon an "anomalous non-contact effect with a delay associated with a pyramidal structure".

By comparing the result for the $\mathrm{ME}$ with that for the p-NME, the existence of the non-contact effect associated with the PS was proved with very high statistical reliability $\left(p=3.51 \times 10^{-6}\right.$, Welch's t-test, two-tails) in the post-1(5h) of the "after meditation period".
The results of the "meditation period" (med-1(0h) and med-2(3h)) in the ME were mostly in agreement with the results of the med-1(0h) and med-2(3h) in the p-NME, where the non-contact effect became zero within the margin of error and was not detected. Before performing the ME, we expected that the noncontact effect toward the bio-sensors would be detected in the "meditation period" in which the meditator is in the PS. However, the results were completely opposite our expectation. We detected the non-contact effect not in the "meditation period" but in the "after meditation period" (post-1(5h), post2(20h)). Therefore, in this paper, we concluded that the term "meditator's non-contact action" rather than the term "meditator's non-contact effect" should be used to describe the phenomenon more correctly. As for the reason, the results of the "meditation period" (med-1(0h) and med-2(3h)) in the ME showed that the meditator did not have the non-contact effect toward the bio-sensors in real time. Instead, the noncontact effect was detected with a delay, which was considered to be associated with the PS. There is a possibility that the meditator's non-contact action may have a real time effect toward the bio-sensors during meditation. But the action has no effect on the gas generation process of the bio-sensors. On the other hand, if the meditator's non-contact action extends to the PS, we speculate that the PS stores the action, and converts it into another action, and the converted action gives an effect to the gas generation process of the bio-sensors. However, by this 
experiment alone, we cannot tell whether the origin of the meditator's non-contact action is "the meditation itself" or "the existence of the meditator as a living object".

Assuming the meditator's non-contact action as the input signal, we calculated the output signal by the transient response of the second order lag element system. As a result, by the time change of the output signal, we were able to approximate the result of an "anomalous non-contact effect with a delay associated with a pyramidal structure" for a period of about 20 days. Moreover, it became clear that this reaction was a phenomenon equivalent to the transient response of the second order lag element system with a damping coefficient $\xi=3.074$, a specific angular frequency $\omega_{\mathrm{n}}=1.286$ and a gain coefficient $\mathrm{K}=1.22$. Therefore, an "anomalous non-contact effect with a delay associated with a pyramidal structure" can be considered to be a transient response phenomenon of the second order lag element system as described below. The meditator's non-contact action is accumulated in the PS, and it is converted into another action. The converted action exercises the non-contact effect toward the bio-sensors.

In the p-NME which was performed approximately 20 days after the standard time (the start of the meditation), the non-contact effect toward the biosensors was not detected. This indicates that the PS itself does not have a function of creating the noncontact effect. Thus, we think it is clear that the noncontact effect on bio-sensors is caused by the presence of the meditator in the PS. Although this is a result we obtained previously (Takagi et al. 2013), this time we have verified it by a different type of experiment, the p-NME. Moreover, we were able to get a better understanding of the non-contact effect. We have shown that the meditator's non-contact action, which does not have a direct non-contact effect, combined with the PS, which does not have a function of creating a non-contact effect by itself, is the cause of the non-contact effect on bio-sensors.

As for the "after meditation period" (post-1(5h), post$2(20 \mathrm{~h})$ ), the non-contact effect was shown to depend on the length of the daytime of the harvest day of the cucumbers. The non-contact effect was detected with summer data, whereas it was not detected with winter data. We attribute this to the fact that the reaction characteristic of the gas generation process in the cucumber changes due to the difference in the daytime length. This probably means that a reaction process, existing only during the summer time, occurs among the various gas generation processes in a cucumber and it is affected by the non-contact effect associated with the PS. By detailed analysis of the difference between summer and winter in the reaction processes to identify the reaction that only exists in summer, we would be able to develop a new physical and/or chemical sensor which can detect the non-contact effect more exactly without depending on bio-sensors, such as cucumbers.

\section{Conclusion}

The following are the especially important results among the various findings we obtained.

We have discovered a new phenomenon an "anomalous non-contact effect with a delay associated with a pyramidal structure" in which the meditator's non-contact action creates a delayed effect over an extended period of time (10 or more days) after the action took place.

In the "after meditation period", the existence of the non-contact effect on bio-sensors with which the pyramidal structure was associated was proved with extremely high statistical reliability $\left(\mathrm{p}=3.51 \times 10^{-6}\right.$, Welch's t-test, two-tails).

We have concluded that the meditator's non-contact action does not have a non-contact effect toward the bio-sensors in real time. Instead, through the intervention with the PS it causes the delayed noncontact effect.

The non-contact effect toward the bio-sensors was not detected during the "meditation period". Instead, it was detected during the "after meditation period", reaching the maximum $20 \mathrm{~h}$ after the standard time. Then, it decreased exponentially, becoming zero within the margin of error. We theoretically studied this anomalous non-contact effect with a delay which is an unexplained phenomenon in modern science. We were able to approximate it by a mathematical formula describing a transient response phenomenon of the second order lag element system, which is a well-known scientific model.

We believe that the report of these discoveries will open a new field in science.

This research was done under the Sakamoto HyperTech Project (SHyP) as a joint study between Aquavision Academy Co., Ltd. (President: Masamichi Sakamoto) and IRI (Chairman of the Board of Directors: Mikio Yamamoto).

\section{Compliance with Ethical Standards}

All procedures followed were in accordance with the ethical standards of the responsible committee on human experimentation (institutional and national) and with the Helsinki Declaration of 1964 and later revision. Informed consent or substitute for it was obtained from meditator for being included in the study.

The authors declare no conflict of interest associated with this manuscript.

The corresponding author had full access to all the data in the study and had final responsibility for the decision to submit for publication. 


\section{References}

1) Atwater F.H. (1997). Accessing anomalous states of consciousness with a binaural beat technology. J. Sci. Explor. 11(3), 263-274

2) Cohen S., \& Popp F.A. (1997). Biophoton emission of the human body. J. Photochem. Photobiol. B: Biol. 40, 187-189. http://dx.doi.org/10.1016/s1011-1344(97)00050-x

3) Grad B. (1976). The Biological Effects of the "Laying on of Hands" on Animals and Plants: Implications for Biology, G. R. Schmeidler, Eds. Parapsychology: Its Relation to Physics, Biology, and Psychiatry (Metuchen, NJ, Scarecrow Press 1976) 76-89.

4) Inaba H. (1997). Photonic sensing technology is opening new frontiers in biophotonics. Optical Review. 4(1A), 1-10. http://dx.doi.org/10.1007/bf02935981

5) Kokubo H., Yamamoto M., \& Kawano K. (2006). Evaluation of non-contact healing using biophotons. J. Intl. Soc. Life Info. Sci. 24(2), 320-327.

6) Kokubo H., Takagi O., \& Yamamoto M. (2009). Development of a gas measurement method with cucumber as a bio-sensor. J. Intl. Soc. Life Info. Sci. 27(2), 200-213.

7) Kokubo H., Koyama S., Takagi O., Kawano K., \& Yamamoto M. (2012). Bio-PK detectivity of fluorescence measurement method: Early spring test. J. Intl. Soc. Life Info. Sci. 30(2), 208-216.

8) Kokubo H., Takagi O., Koyama S., \& Yamamoto M. (2011) Discussion of an approximated equation for special distribution of controlled healing power around a human body. J. Intl. Soc. Life Info. Sci. 29(1), 23-34.

9) Kokubo H., Koyama S., \& Takagi O. (2010). Relationship between biophotons and gases generated from cucumber pieces. J. Intl. Soc. Life Info. Sci. 28(1), 84-94.

10) Kokubo H., Takagi O., \& Koyama S. (2010). Application of a gas measurement method - Measurement of ki fields and non-contact healing-. J. Intl. Soc. Life Info. Sci. 28(1), 95-112.

11) Parkhomtchouk D., Hirasawa M., \& Yamamoto M. (2000). Low noise system with optical cable probe for ultra-weak photon emission measurements from living matter. J. Intl. Soc. Life Info. Sci. 18(1), 109-116.

12) Takagi O. et al., (2013). Meditator's non-contact effect on cucumbers. Intl. J. Phys. Sci. 8(15), 647-651.

13) Yamamoto M. et al. (2000). Study on analyzing methods of human body functions using various simultaneous measurements. J. Intl. Soc. Life Info. Sci. 18(1), 61-80.

14) Yamamoto M. et al. (2002). Are there any effects other than suggestion in external Qi. J. Intl. Soc. Life Info. Sci. 20(2), 317-342.

15)

\section{Meditation Experiment : ME}

\section{"Before and After Meditation Periods" Meditator absence}

pre-1 (-16h) pre-2 (-3h) post-1 (5h) post-2 (20h)
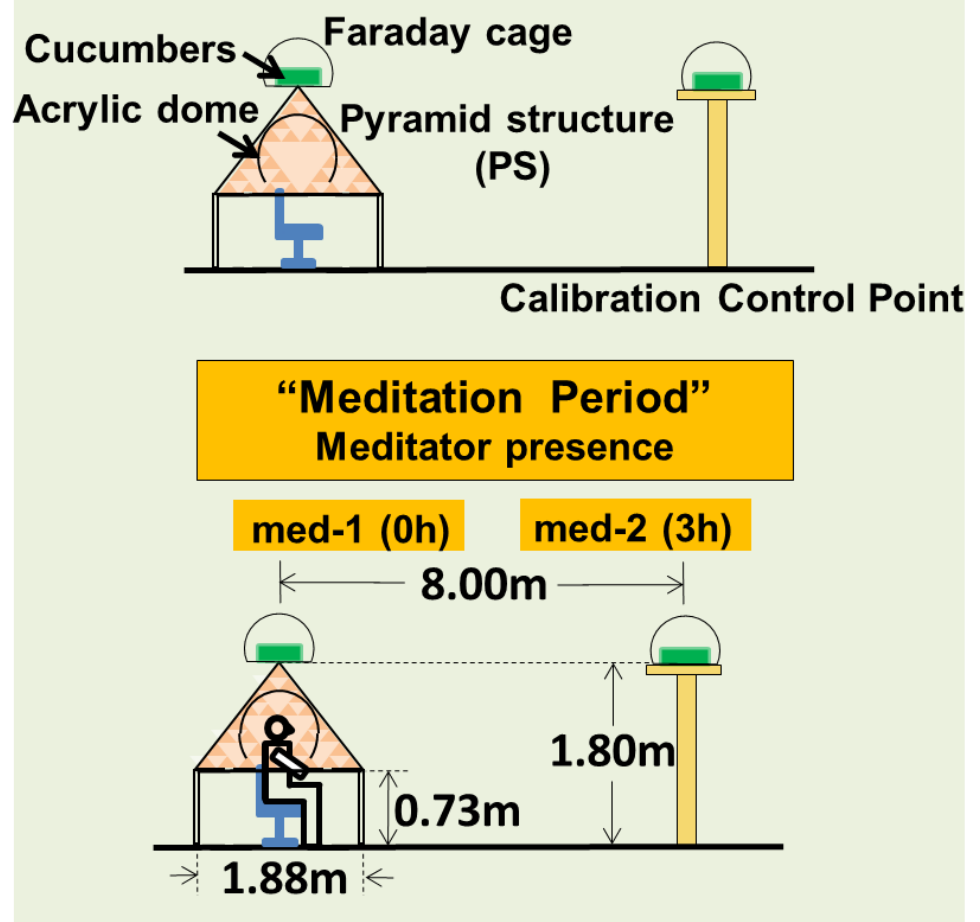

Fig. 1. Setup for the ME

In the ME, during the "before and after meditation periods", the meditator was not present. The "before meditation period" consisted of two parts, pre-1($16 \mathrm{~h})$ and pre-2(-3h), while the "after meditation period" also consisted of two parts, post-1(5h) and post-2(20h). During the "meditation period", the meditator was inside the PS and the "meditation period" consisted of two parts, med-1(0h) and med2(3h). 


\section{(a) Meditation Experiment : ME}

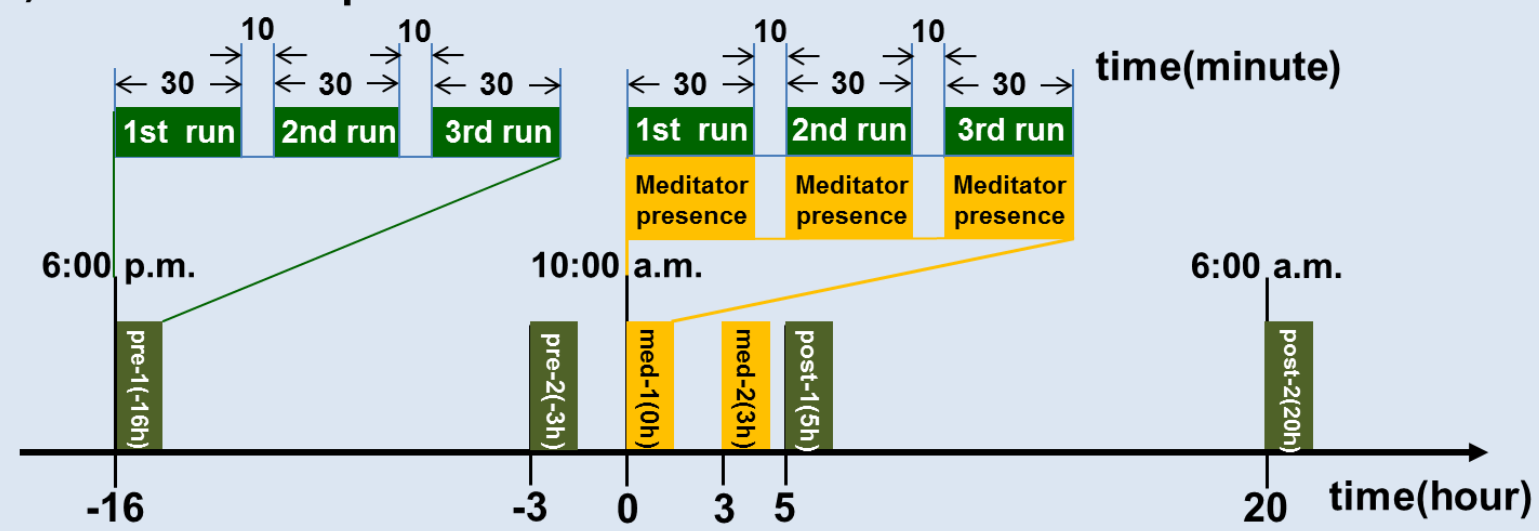

(b) Non-Meditation Experiment : NME \& Pure Non-Meditation Experiment : p-NME

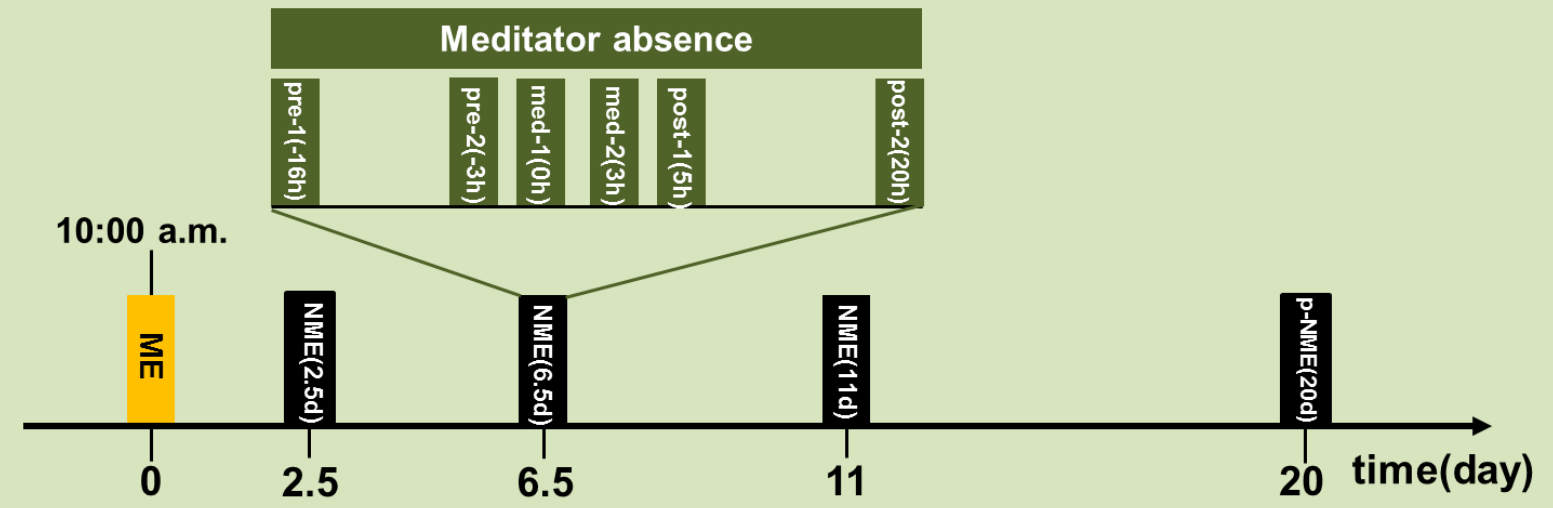

Fig. 2. Time schedules for the ME and the NME

Figure 2(a) shows the time sequence (in hours) of the ME with six parts from pre-1(-16h) to post-2(20h). Here, one part consisted of three runs. Figure 2(b) shows the time sequences (in days) of the NME and the p-NME. The NME was performed a few days after the ME. The only difference between them was whether or not the meditator was present, all other factors were the same. The p-NME was an NME performed about 20 days or more after the ME. 


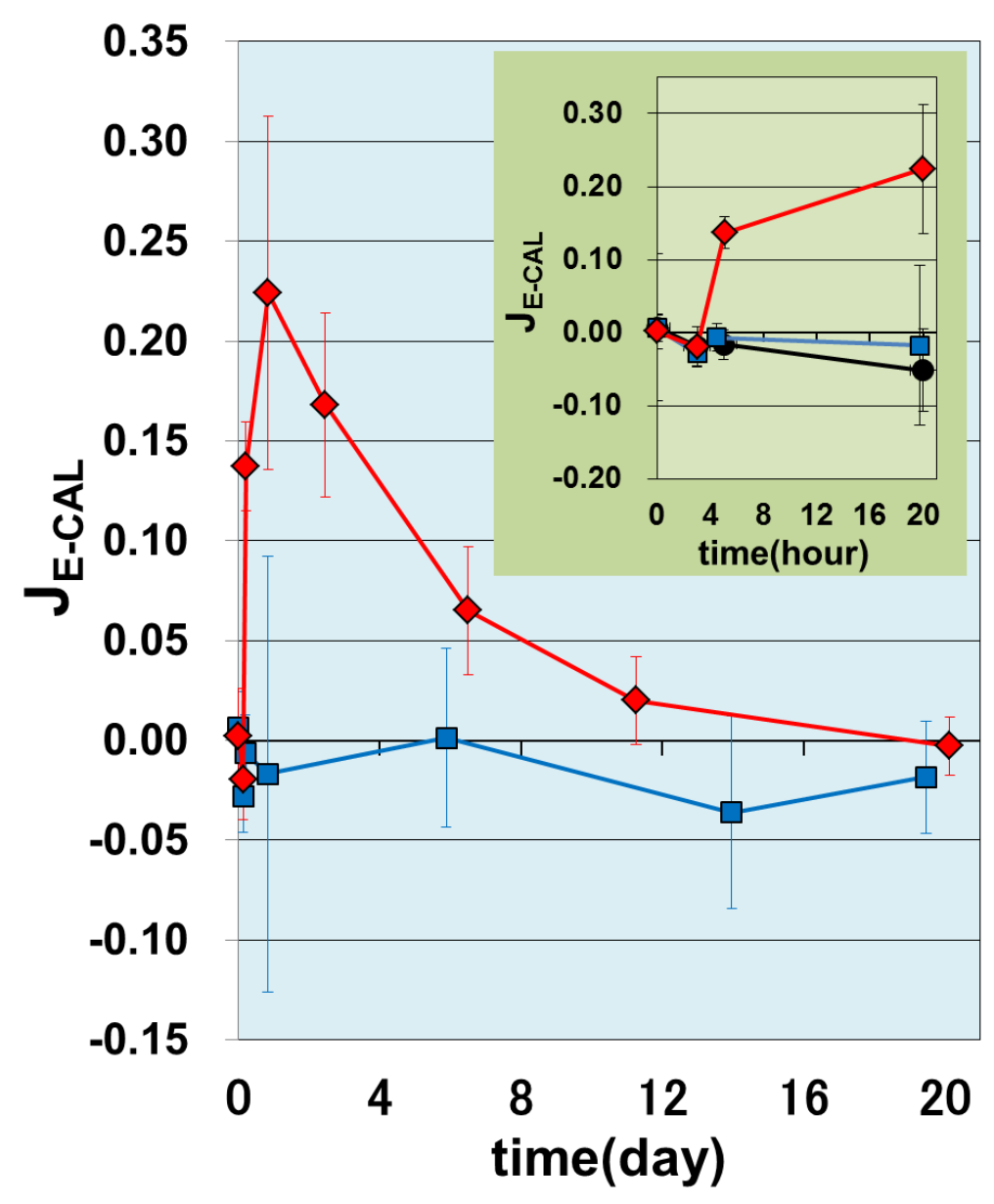

Fig. 3. Experimental results of anomalous non-contact effect with a delay

Figure 3 shows the results for the four parts of the ME (med-1(0h), med-2(3h), post-1(5h) and post2(20h)) and for the NME done a few days after the standard time 0 . For the summer data, the detected value $\mathrm{J}_{\mathrm{E}-\mathrm{CAL}}$ for the "meditation period" (med-1(0h) and med-2(3h)) was zero within the margin of error. 5 $\mathrm{h}$ after the standard time (post-1(5h)), it started to increase, reached the maximum at $20 \mathrm{~h}$ (post-2(20h)) and then decreased exponentially after 10 or more days. It became almost zero about 20 days after the standard time. In contrast, for the winter data, $\mathrm{J}_{\mathrm{E}-\mathrm{CAL}}$ values were zero within the margin of error for all the data points, i.e. med-1(0h), med-2(3h), post-1(5h) and post-2(20h) and also the NME (6 days and 14 days after the standard time) and p-NME (19.5 days after the standard time). Each error bar indicates the standard error. The inset shows the results of four parts, med-1(0h), med-2(3h), post-1(5h) and post$2(20 \mathrm{~h})$ for both the $\mathrm{ME}$ and the p-NME. By comparing the p-NME data with the ME summer data for post-1(5h), we were able to prove the existence of the non-contact effect associated with the PS with very high statistical reliability $\left(p=3.51 \times 10^{-6}\right.$, Welch's t-test, two-tails). 


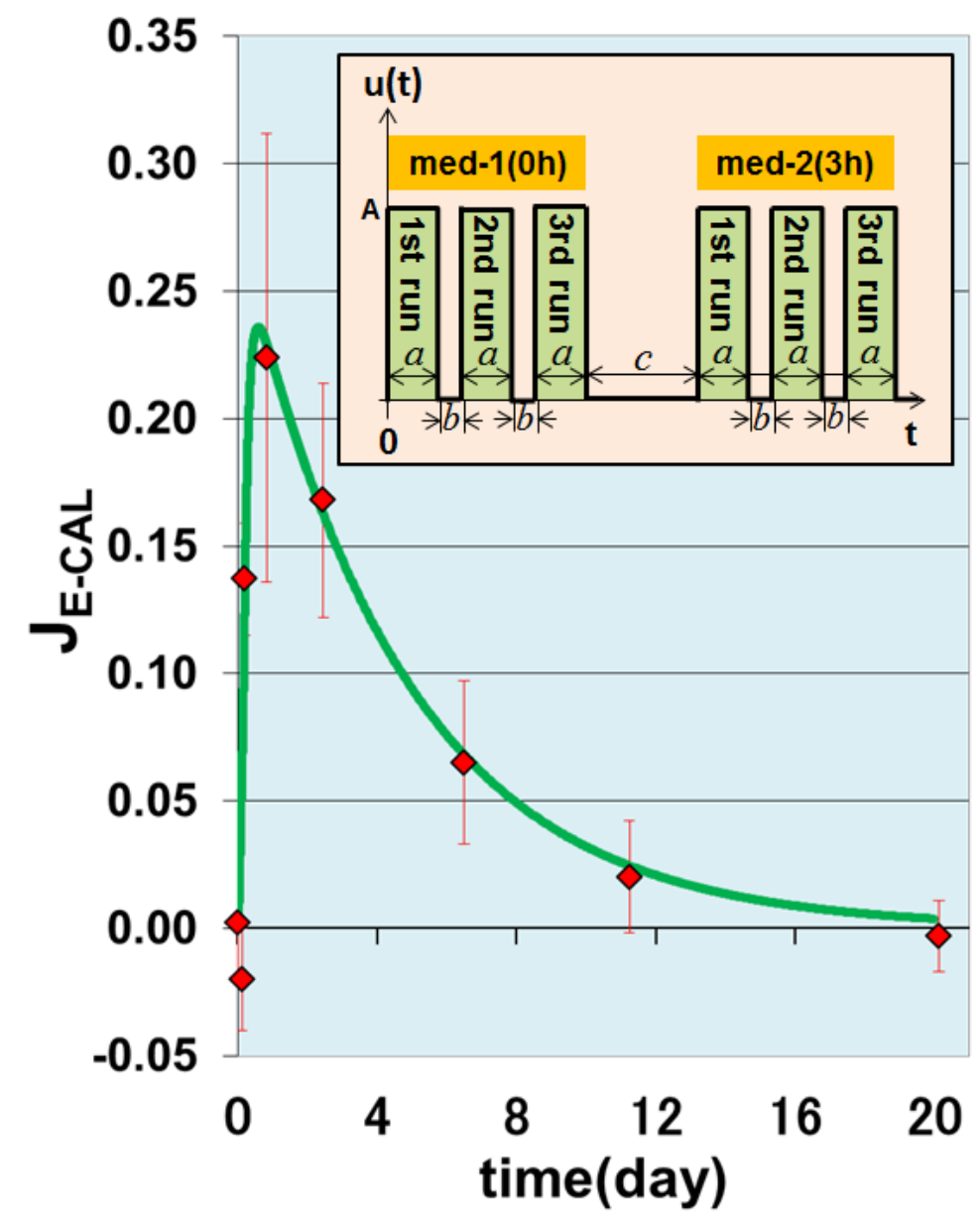

Fig. 4. Experimental results and theoretical curve for anomalous non-contact effect with a delay

Figure 4 plots the summer data results of Figure 3 together with the theoretical curve results. Here, $A=8, K=1.22$, $(\tau 1$, $\tau 2)=(4.65,0.13)$ or $(0.13,4.65)$. This theoretical curve corresponded to a transient response phenomenon of the second order lag element system with a damping coefficient $\xi=3.074$, a specific angular frequency $\omega_{\mathrm{n}}=1.286$ and a gain coefficient $\mathrm{K}=1.22$. The inset shows the shape of the input signal $u(t)$. Here, $a=30$ min=0.0208 days, $b=10$ $\min =0.0069$ days, $c=70 \mathrm{~min}=0.0486$ days. The theoretical curve was in good agreement with the actual experimental data for the anomalous non-contact effect with a delay. 